\title{
DELPHIC SEMIGROUPS
}

\author{
BY DAVID G. KENDALL
}

Communicated by M. Loeve, July 25, 1966

A delphic semigroup shall be a topological commutative semigroup which is Hausdorff and possesses a neutral element and satisfies the three conditions $(\mathrm{A}-\mathrm{C})$ below. In formulating these we require some terminology: a triangular array is a system $u(i, j)(i=1,2, \cdots$; $j=1,2, \cdots, i)$ of elements of the semigroup; the $i$ th marginal product is the element

$$
u(i, 1) u(i, 2) \cdots u(i, i) ;
$$

an array is said to converge to an element $u$ when the $i$ th marginal product converges to $u$; an element is said to be infinitely divisible when it possesses a $k$ th root for each $k \geqq 2$.

(A) There exists a continuous homomorphism $\Delta$ from the semigroup into the additive semigroup of nonnegative reals, such that $\Delta(u)=0$ if and only if $u$ is the neutral element.

(B) The set $\left\{u^{\prime}: u^{\prime} \mid u\right\}$ of factors of any given element $u$ is compact.

(C) If a triangular array converges to $u$, and if the array satisfies the condition $\Delta(u(i, j)) \rightarrow 0$ as $i \rightarrow \infty$ uniformly for $1 \leqq j \leqq i$, then $u$ is infinitely divisible.

As a nontrivial example we mention here only the multiplicative semigroup of positive renewal sequences; for the complete details of this and other examples, as well as for the proofs of the following theorems, reference should be made to [1] and [2].

The first property of a delphic semigroup is that every infinitely divisible element $u$ can be represented as a limit as in (C). Next, it can be shown that the elements of such a semigroup can be partitioned into three exhaustive and mutually exclusive classes; the elements in the first class are indecomposable; those in the second class are decomposable but possess an indecomposable factor; those in the third class are infinitely divisible and possess no indecomposable factor. Finally it can be shown that an arbitrary element of such a semigroup possesses at least one representation in the form

$$
u=v(1) v(2) \cdots w,
$$

where the $v$ 's are indecomposable and $w$ is infinitely divisible and possesses no indecomposable factor. There are not more than countably many $v$ 's; there may be none. 
The formulation and study of this axiomatic system was suggested by the discovery [1] that Khintchine's factorization theorems for the convolution semigroup of probability distributions on $R$ can be extended to the semigroup of renewal sequences, among others.

\title{
REFERENCES
}

1. D. G. Kendall, Renewal sequences and their arithmetic, Proc. Loutraki Symposium on Probabilistic Methods in Analysis, Springer, Berlin, (to appear in 1966).

2. - Delphic semi-groups, infinitely divisible regenerative phenomena, and ihe arithmetic of p-functions, (in preparation).

Churchill College, Cambridge, England.

\section{AN ALGEBRAIC CONJUGACY INVARIANT FOR MEASURE PRESERVING TRANSFORMATIONS ${ }^{1}$}

\author{
BY WILLIAM B. ARVESON
}

Communicated by P. R. Halmos, August 1, 1966

Let $T$ be an invertible, ergodic, measure-preserving transformation of a separable, nonatomic probability space $(X, B, m)$, and let $U$ be the induced unitary operator acting in $L^{2}(X, B, m)$. Let $Q(T)$ be the Banach algebra generated by the multiplication algebra and the nonnegative powers of $U$. It is shown that, if $S$ is another such transformation, then $S$ and $T$ are conjugate if, and only if, $Q(S)$ and $a(T)$ are unitarily equivalent. Thus, the conjugacy problem for ergodic transformations is equivalent to multiplicity theory for the algebras $a(T)$. While much remains to be learned about these operator algebras, similar ones have been studied in [5] and [1]. Finally, $Q(T)$ can be realized concretely as an algebra of operator-valued analytic functions in the unit disc.

In $\$ 2$ we describe generalizations of the $C^{*}$-algebra constructed in $\$ 1$; it turns out that pathology appears as soon as the group involved fails to be amenable, and only in that case.

Full details and further developments will appear elsewhere.

1. The algebras $a$ and $B$. For definiteness, we assume all transformations act on the unit interval, are Borel measurable, and pre-

1 This research was supported by the U. S. Army Research Office (Durham). 